\title{
Functional neuroimaging in subjective cognitive decline: current status and a research path forward
}

\author{
Raymond P. Viviano ${ }^{1,2}$ and Jessica S. Damoiseaux ${ }^{1,2^{*}}$
}

\begin{abstract}
Subjective cognitive decline is a putative precursor to dementia marked by perceived worsening of cognitive function without overt performance issues on neuropsychological assessment. Although healthy older adults with subjective cognitive decline may function normally, perceived worsening may indicate incipient dementia and predict future deterioration. Therefore, the experience of decline represents a possible entry point for clinical intervention. However, intervention requires a physical manifestation of neuroabnormality to both corroborate incipient dementia and to target clinically. While some individuals with subjective cognitive decline may harbor pathophysiology for specific neurodegenerative disorders, many do not display clear indicators. Thus, disorder-agnostic brain measures could be useful to track the trajectory of decline, and functional neuroimaging in particular may be sensitive to detect incipient dementia and have the ability to track disease-related change when the underlying disease etiology remains unclear. Therefore, in this review, we discuss functional neuroimaging studies of subjective cognitive decline and possible reconciliations to inconsistent findings. We conclude by proposing a functional model where noisy signal propagation and inefficient signal processing across whole-brain networks may lead to the subjective experience of decline and discuss future research directions guided by this model.
\end{abstract}

Keywords: Subjective cognitive decline, Functional neuroimaging, Connectivity

\section{Introduction}

Subjective cognitive decline (SCD) is a putative precursor to dementia marked by professed worsening of cognitive functioning, in any domain, without explicit performance issues on neuropsychological assessment. Precise definition and labeling of the concept have evolved over the past decades, with an international working group recently coming to a consensus to help spur data consistency [1]. Briefly, "subjective" denotes personal experience and can be orthogonal to objective

\footnotetext{
* Correspondence: damoiseaux@wayne.edu

'Department of Psychology, Wayne State University, 5057 Woodward Ave. 7th Floor Suite 7908, Detroit, MI 48201, USA

${ }^{2}$ Institute of Gerontology, Wayne State University, 87 E. Ferry St., Detroit, Ml 48202, USA
}

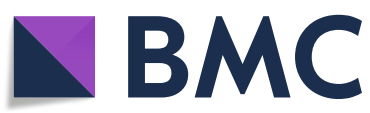

(c) The Author(s). 2020 Open Access This article is licensed under a Creative Commons Attribution 4.0 International License, which permits use, sharing, adaptation, distribution and reproduction in any medium or format, as long as you give appropriate credit to the original author(s) and the source, provide a link to the Creative Commons licence, and indicate if changes were made. The images or other third party material in this article are included in the article's Creative Commons licence, unless indicated otherwise in a credit line to the material. If material is not included in the article's Creative Commons licence and your intended use is not permitted by statutory regulation or exceeds the permitted use, you will need to obtain permission directly from the copyright holder. To view a copy of this licence, visit http://creativecommons.org/licenses/by/4.0/ The Creative Commons Public Domain Dedication waiver (http://creativecommons.org/publicdomain/zero/1.0/) applies to the data made available in this article, unless otherwise stated in a credit line to the data. may not notice worsening cognitive ability but perform poorly on assessment, while an individual with perception of decline may perform within a normal range [2]. Although not a concurrent indicator of objective decline, evidence suggests SCD can predict future cognitive deterioration and may demarcate incipient dementia [3-7]. The term "cognitive" implies that perceived worsening may apply to any domain, not just memory function. This ensures that research efforts capture trajectories from SCD to dementia when initial symptoms are unrelated to memory. Many earlier definitions focused on perceived memory impairment [8]; however, cognitive is a more beneficial label than memory as individuals may misreport problems in other cognitive domains as 
memory problems. Finally, decline reflects a progressive experience of cognitive worsening rather than an acutely caused stable impairment. On an individual basis, decline may relate to healthy or pathological aging; regardless, the consensus label has shifted to decline from impairment to reflect a progressive nature-possibly due to underlying neurodegeneration.

The memory component of SCD has been the dominant focal point of prior research, and early data collection efforts emphasized memory deficit by determining SCD through a single question or a set of questions about perceived memory [8]. In addition, earlier definitions placed SCD as a stage directly before mild cognitive impairment on the healthy aging to Alzheimer's disease (AD) continuum [9]. However, SCD has multiple etiologies and potential outcomes [1]; therefore, classification as a symptom or risk factor of incipient dementia may be more appropriate. Nevertheless, many reports discuss SCD within an AD framework. According to the modern research definition by the National Institute on Aging and the Alzheimer's Association [10], Alzheimer's disease is a designation for individuals who exhibit pathological amyloid- $\beta$ and tau deposits, determined either through positron emission tomography or cerebrospinal fluid evaluation. Although discussion of SCD biases towards a precursor to $\mathrm{AD}$, and $\mathrm{SCD}$ associates with future dementia development $[1,3]$, associations between SCD and amyloid- $\beta$ and tau burden are mixed [11-18]; thus, an Alzheimer's-centric approach could misguide interpretation of SCD data. Although AD may be the most common dementia etiology [19], and individuals with $\mathrm{SCD}$ may receive an $\mathrm{AD}$ diagnosis over time, SCD possibly serves more accurately as a branching point between cognitively intact aging and a myriad of potential outcomes, including various dementias but also, commonly, recovery to a subjectively unimpaired state. Indeed, for many, SCD may be a temporary event.

As there is no one-to-one mapping between SCD and preclinical $\mathrm{AD}$, amyloid- $\beta$ and tau burden evaluation alone are not the optimal strategies to characterize SCD. Furthermore, prior analyses and models propose that functional brain network communication may break down prior to amyloid- $\beta$ accumulation [20,21], suggesting that functional brain alterations are sensitive metrics for early change. Therefore, measures that are nonspecific to disease outcome may be more appropriate to evaluate SCD than disease-specific indicators and could identify patterns of brain structure or function that discriminate between individuals who remain cognitively unimpaired from individuals who develop dementia at a later time. Furthermore, functional neuroimaging may be a viable method for tracking change in SCD as evidenced by previous longitudinal work showing change related to aging and neurodegenerative disease [22-24].
Therefore, here, we review the functional neuroimaging literature in SCD, propose a functional model of SCD where differences in signal processing across wholebrain networks may lead to the experience of cognitive decline, and suggest future research efforts to test the model. This model refers to individuals who experience SCD more likely due to nascent neurodegeneration than other causes and are at greater risk of developing dementia rather than individuals who exhibit cognitive maintenance or for whom SCD is a temporary event.

\section{Search and review methodology}

We searched the PubMed and Web of Science Core databases for articles evaluating functional characteristics of the brain in SCD. Title, keyword, and abstract search terms included either subjective cognitive decline, subjective executive decline, subjective memory impairment, subjective cognitive impairment, subjective cognitive concerns, or subjective memory concerns. We then narrowed the results of the pooled broad searches with functional, connectivity, activity, magnetic resonance (fMRI), infrared spectroscopy, electroencephalography (EEG), and magnetoencephalography (MEG) as additional terms. After sifting through titles and abstracts, we identified 38 relevant articles. Table 1 provides an overview of the methods and sample characteristics for these prior analyses; it also provides more comprehensive overviews of specific results than we address in the body of the text. We excluded articles involving an intervention (e.g., diet or resveratrol), where SCD was a continuous variable measured within a patient population (e.g., subjective decline in Parkinson's disease), where SCD was unrelated to aging (e.g., cancer, fatigue, multiple sclerosis), where there was no control group without cognitive concerns or no continuous measure of $\mathrm{SCD}$, and when the methods did not include functional imaging.

As mentioned previously, SCD outcomes are varied, with some individuals reverting to a subjectively unimpaired state while others may develop dementia. Furthermore, there are additional factors that could influence perception and report of perceived cognitive decline, such as personality or depression [62-68]. However, for the analyses considered in this review, many research groups recruited samples from memory clinics, adhered to guidelines proposed by an international working group [1], and accounted for potential confounds either in recruitment or during data analysis. Therefore, we are confident that a synthesis of this literature reflects functional brain characteristics of SCD for individuals who may harbor incipient neurodegeneration and are at greater risk of developing dementia, and thus interpret the results within that assumption. 


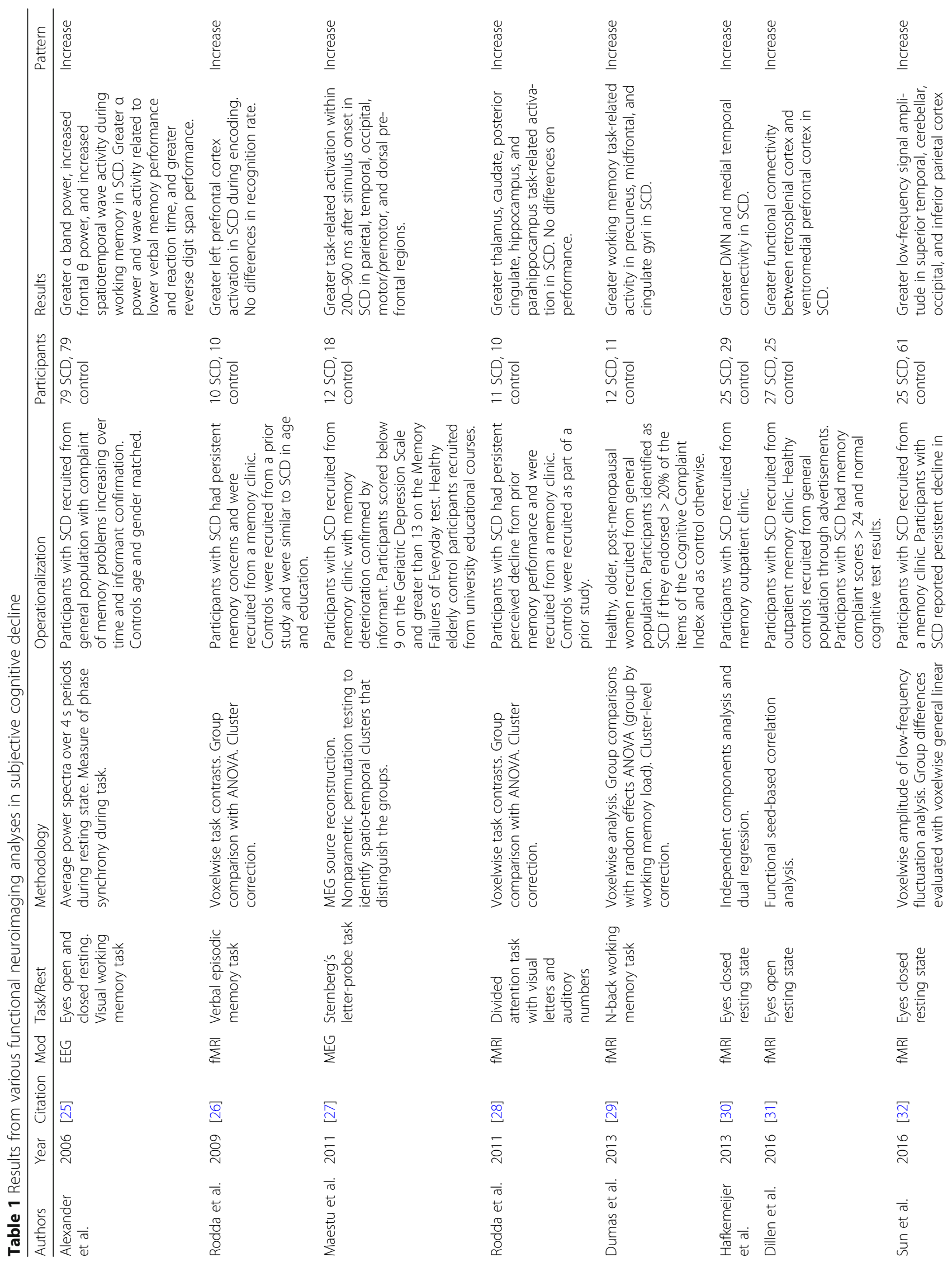




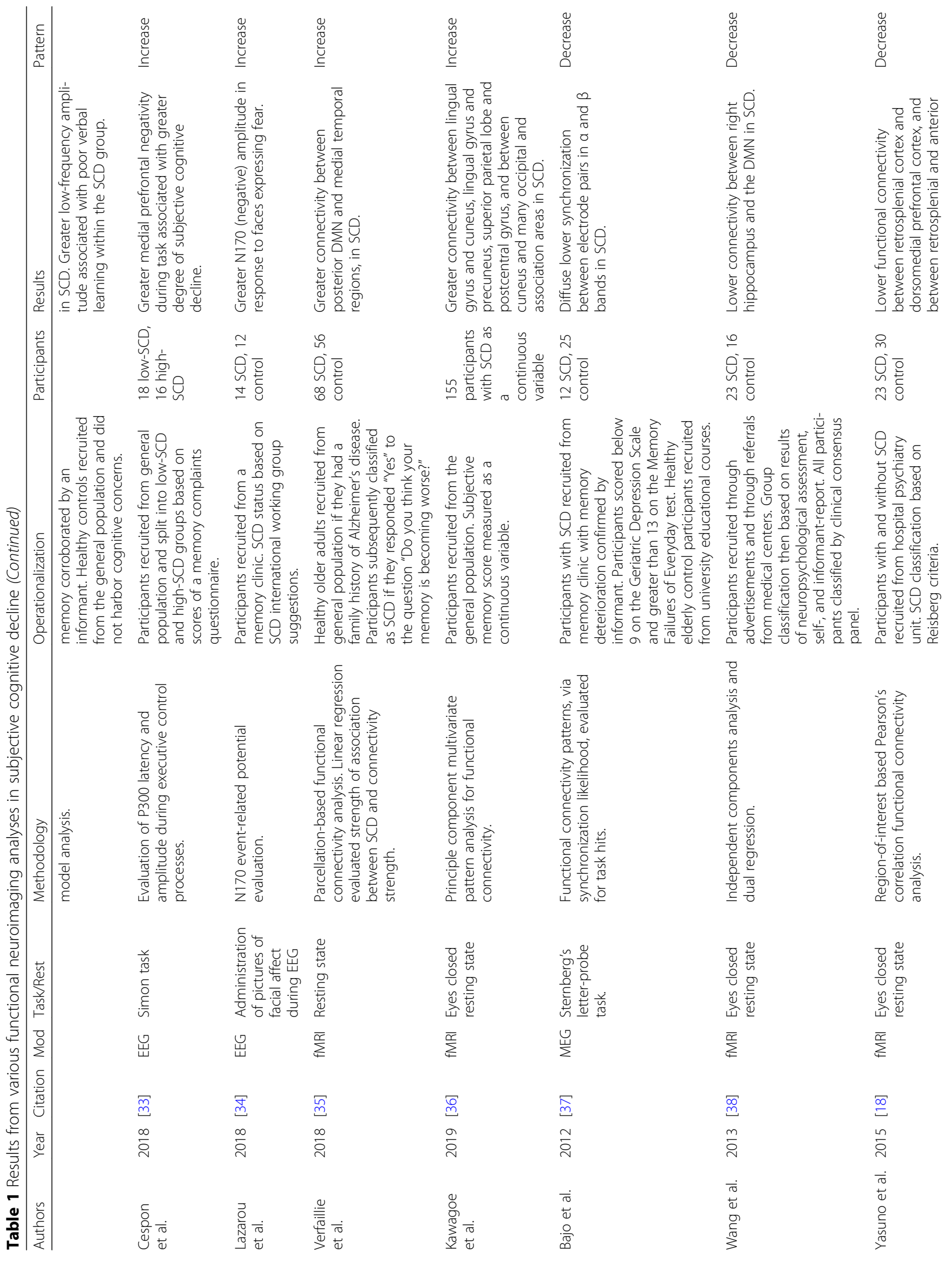




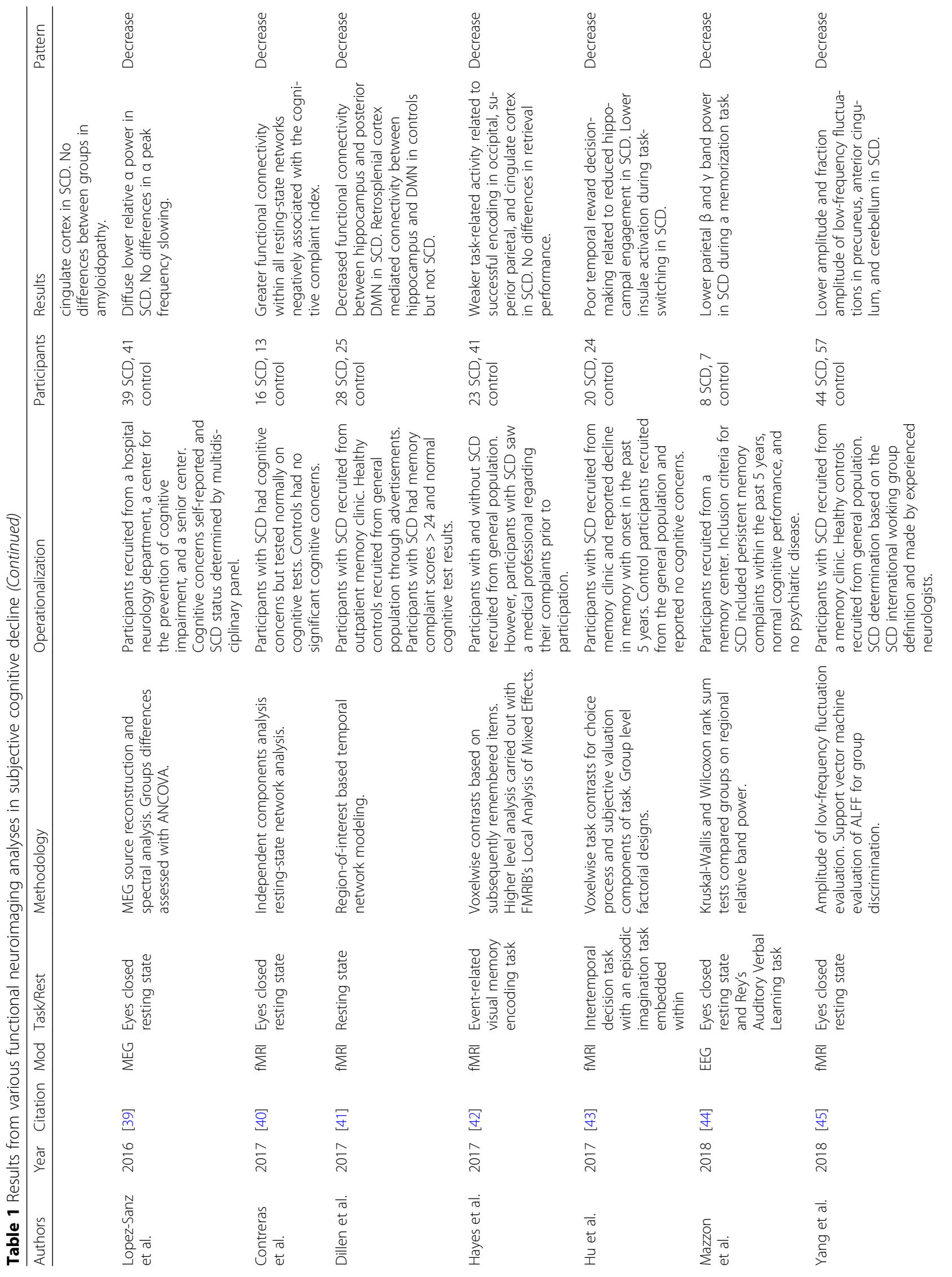




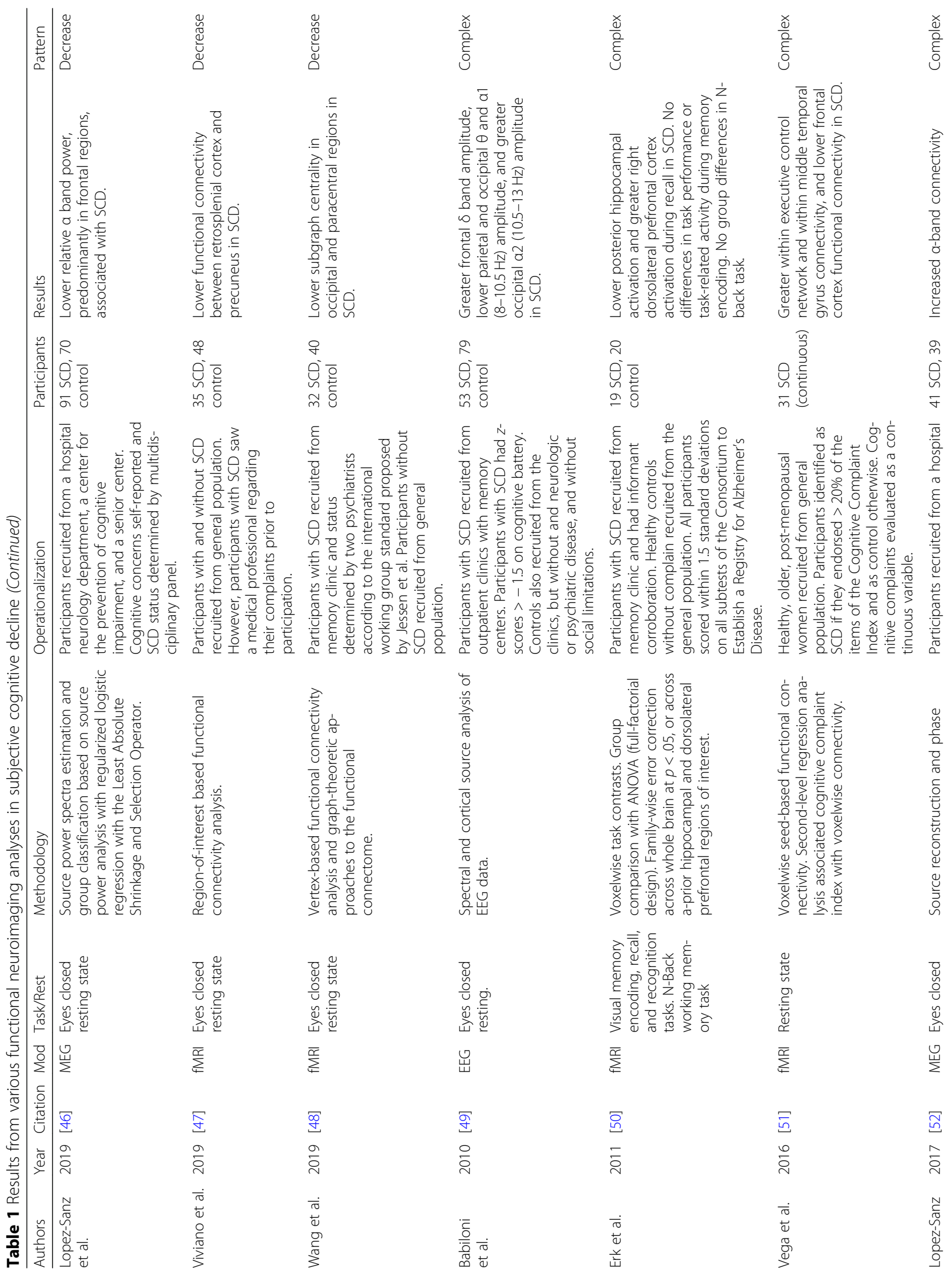




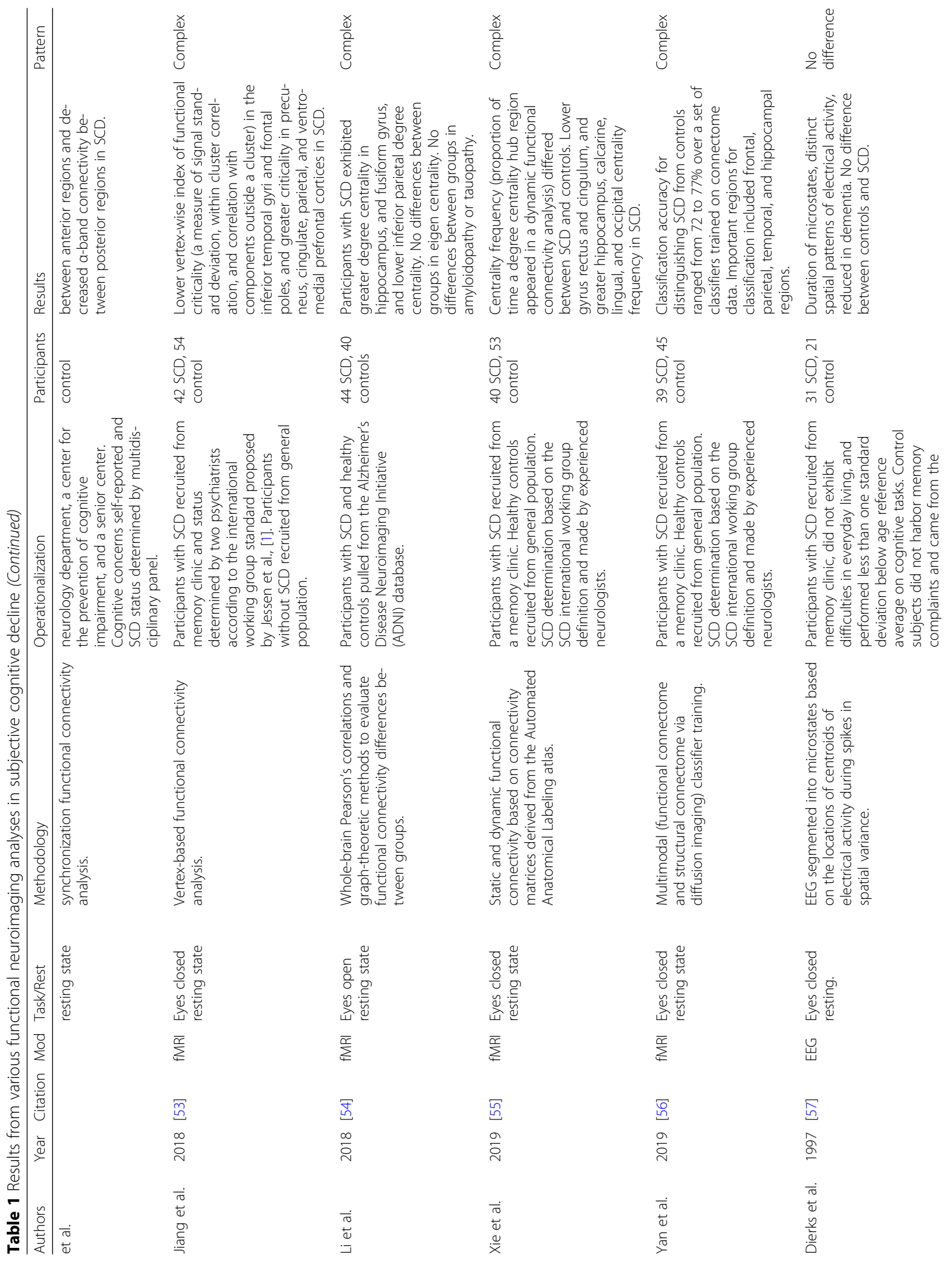




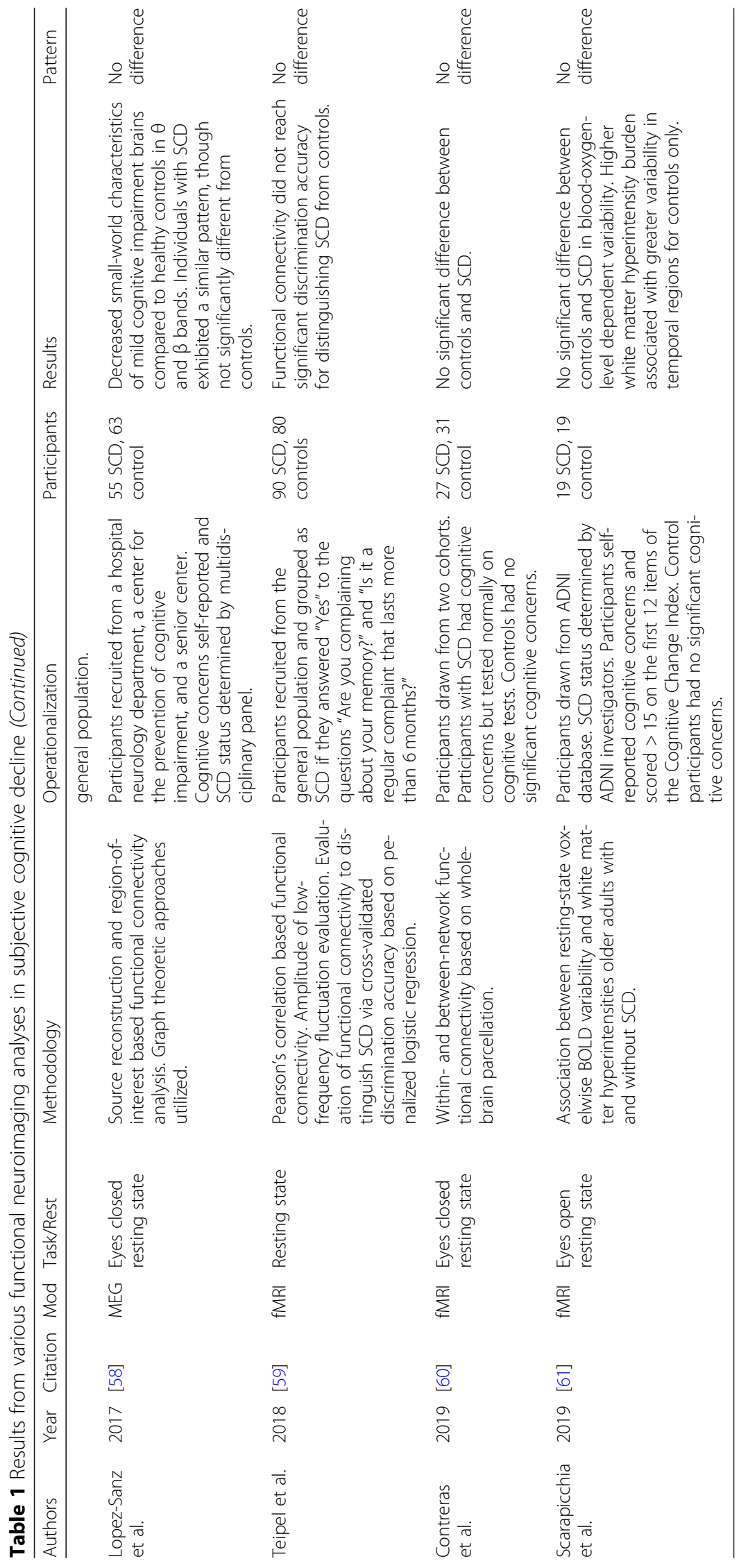




\section{Functional neuroimaging in subjective cognitive decline}

Task-related brain activity in subjective cognitive decline

Previous fMRI analyses that examined memory processing in older adults have observed lower hippocampal and greater dorsolateral prefrontal cortex task-related activity during memory retrieval, as well as greater task-related activity in dorsolateral prefrontal cortex and lower activity in superior parietal, cingulate, and occipital cortex during memory encoding in individuals with $\operatorname{SCD}[26,42,50]$. These results suggest that SCD may arise from processing aberrations underlying either memory encoding or retrieval. As different analyses uncovered encoding or retrieval group differences, the ability to efficiently access stored information may diminish first for some while the ability to efficiently encode information may deteriorate earliest for others. This suggests that episodic memory encoding and retrieval processing alterations are independently sufficient for the subjective experience of decline, but neither are necessary.

In addition to episodic memory, fMRI analyses have also identified group differences in functional activity during working memory and other executive functions in SCD. Prior analyses suggest greater working memoryrelated activity in precuneus, middle frontal gyrus, and cingulate gyrus [29], and greater divided attentionrelated activity in thalamus, caudate, posterior cingulate, hippocampus, and parahippocampus [28] in individuals with SCD. Moreover, greater EEG alpha band power across electrodes, greater frontal theta power, and greater spatiotemporal wave activity during working memory have been observed in older adults with SCD, with greater alpha power and wave activity relating to lower verbal memory performance and reaction time, and greater reverse digit span performance [25]. Finally, greater MEG post-stimulus-related activity has been noted in older adults with SCD in parietal, temporal, occipital, motor, and dorsal prefrontal during a working memory task [27]. Overall, these analyses provide evidence that neural information processing disruptions in SCD do not just relate to memory but may also extend to executive functions.

Though many analyses have evaluated functional brain aberrations related to memory and executive functions, these processing disturbances may not be the sole drivers of SCD. Previously observed lower insula activation in individuals with SCD during a task that involved switching between imagination and temporal decisionmaking suggests decreased ability to control engagement of the default mode and executive control networks in SCD [43]. The default mode network is involved in episodic memory, theory of mind, and future planning [69], while the executive control network is involved in working memory, attention, and other executive functions [70, 71]. Lower insula activation during task switching [43], however, suggests that SCD may relate to inefficient salience network functioning, as the insulae are part of a network observed to send control signals to the default mode and executive control regions to modulate their engagement relative to internal and external task demands [72]. Thus, it is possible that the circuitry involved in memory and executive functions may operate efficiently in some individuals with SCD, but the ability to properly recruit different circuits at appropriate times diminishes.

Overall, tasked-based analyses suggest that cognitive processing alterations exist in individuals with SCD, but precise and accurate synthesis of the results remains difficult. Lower hippocampal task-related activity during retrieval [50] and decreased occipital and default mode network task-related activity during encoding [42] could either indicate local processing inefficiencies in the observed regions or indicate decreased network signaling to those regions during memory processing in SCD. This interpretation is based on multimodal imaging research that indicates that neuronal contributions to the blood-oxygen-level dependent (BOLD) response reflect metabolic demands of synaptic activity [73], which suggests that regional signal reflects local computation and processing of distal afferent input. This interpretation of the BOLD response also contextualizes observations of greater task-related activity in frontal regions during encoding or retrieval $[26,50]$, which could reflect either greater processing within regional microcircuits, or greater processing of afferent input to frontal regions. Greater processing of afferent input could reflect functional compensation [74]. Here, the neurocognitive Scaffolding Theory of Aging and Cognition would posit that cognitive ability remains relatively stable through aging despite neuronal declines, and the continuous recruitment of additional neuronal circuits, which may manifest as functional activity increases via neuroimaging analysis, may be responsible for stable cognitive ability through healthy aging and early disease states. Thus, functional activity increases in frontal regions in SCD could represent compensatory recruitment (more processing of afferent input) to aide processing deficiencies in circuits with neural insults. Greater executive taskrelated activity and greater alpha band power in individuals with SCD [25, 27-29] could also indicate explicit strategy use in response to self-knowledge of decline, rather than compensatory regional recruitment outside of cognitive control. Though, greater alpha band power in older adults with SCD under cognitive load could also reflect more automatic 
compensatory processing to nascent decline, an interpretation supported by increased band power relating to better executive performance [25].

However, another valid interpretation of greater regional task-related activity in SCD could be that computational failure in a circuit leads to a shifting of processing loads and computation to other regions. While the distinction between compensatory recruitment and shifting processing load is subtle, this reframing of interpretation would fit the Cascading Network Failure model of AD [20]. Furthermore, although compensatory processing is a common chorus across discussion sections, increased task-related activity could also reflect inefficient processing. Briefly, decreased network segregation or age-related functional dedifferentiation $[75,76]$, which could reflect less economical neural function in aging where maintenance of cognitive abilities may require greater BOLD signal $[77,78]$, could be accelerated in SCD and reflect inefficient information processing and noisy signal propagation across brain networks. Synaptic disruption, supported by observations of structural atrophy in SCD [79-86], could also result in unrefined noisy signal. Therefore, it could be that the increased task-related activity and alpha band power during executive functions reflects increased processing demands in individuals with SCD, possibly from inefficient signal processing and system noise, rather than compensatory mechanisms. For example, increased spatiotemporal wave activity [25] could indicate lower stability of distinct states of information processing, and greater alpha band power could indicate increased processing demands of attention without necessarily offering a compensatory advantage. Negative associations of alpha band power with verbal memory performance and reaction time [25] could support this interpretation.

Overall, the exact interpretations of complex patterns of increased and decreased task-related neural activity remain up for debate. However, existing theories and models of cognitively unimpaired aging and dementia development, e.g., the Scaffolding Theory of Aging and Cognition [74], the Dedifferentiation Theory of Aging [76], or the Cascading Network Failure Model of AD [20], offer valid interpretations for comparing the functional brains of older adults with and without SCD. There is also room for a general model that unifies concepts across these theories and models to explain functional alterations of the brain in SCD. Compensatory neural recruitment, non-compensatory network dedifferentiation, cascading network failures and processing load shifts, noisy signal propagation, and inefficient network signal processing could all occur in the same brain; one mechanism could influence observed neural activity in one region while a different mechanism affects the activity in another region.

\section{Brain network functional connectivity in subjective cognitive decline at wakeful rest}

Variability and inconsistencies across the resting-state functional connectivity literature in SCD have resulted in a challenging picture to decipher. Some groups have reported greater connectivity, mainly within and between default mode network and medial temporal regions, in older adults with SCD [30, 31, 35, 36]. However, many groups have also observed lower connectivity between these regions in individuals with SCD [18, 76-79]. Others have reported no difference from control [60], or intricate patterns of greater and lower functional connectivity in individuals with SCD [51-55], suggesting that a complex reorganization of processing responsibilities across network nodes may occur. Multifaceted patterns of increased and decreased connectivity between regions could reflect breakdown in processing within a subnetwork that shifts processing load to more intact components of the broader network. Diffuse and frontally localized MEG alpha band power reductions have also been noted in older adults with SCD [39, 46], without group differences in the small world properties of theta and beta bands [58]. Moreover, lower subgraph centrality (a measure of weighted, closed walks starting and ending at a node, representing mid-scale connectivity) has been observed in occipital and paracentral regions in older adults with SCD [48].

Though the functional connectivity results appear incongruous, they do suggest that connectivity abnormalities occur in SCD that could represent information processing inefficiencies and could demarcate incipient dementia. The implications of elevated functional connectivity in SCD [30, 31, 35] are unclear but could reflect compensatory intrinsic signaling resulting from gray matter atrophy or other neural insult. This could reflect a neurocognitive scaffolding mechanism for maintaining stable internal mentation and memory functioning [74]. However, increased connectivity does not always confer greater cognitive performance and may reflect a shift in network properties that underlies poor memory performance [87]. Increased processing demands for internal mentation and memory at rest could arise due to noisier information traveling through memory systems [77]. Increasing regional metabolic demands from processing noisy information could serve as a mechanism that induces further neurodegenerative decline. Eventually, this neurodegenerative pressure could shift elevated functional connectivity early in SCD to decreased functional connectivity later in SCD.

Lower functional connectivity between default mode and medial temporal structures in SCD [18, 37, 38, 41, 47] could either reflect cohort differences compared to analyses noting greater functional connectivity in SCD 
[30, 31, 35, 36] or represent a later phase of SCD close to mild cognitive impairment conversion marked by decreased signaling capability between regions. While involved in episodic memory, cortical midline regions of the default mode network also mediate self-referential processing [69, 88]. Therefore, reduced connectivity could indicate either decreased computation related to self-awareness of memory processing or decreased memory processing directly. Furthermore, as cortical midline structures, hippocampus, and parahippocampus compose a memory system import for accessing episodic memories [89], and as decreased posterior hippocampal activation during memory recall has been noted in older adults with SCD [50], the aforementioned task and resting-state results together provide parallel evidence that issues in memory access processing are either a common or a sufficient component of SCD.

The confounding variables responsible for discrepancies in the results remain unclear; however, a few differences across samples could help explain the results. For example, [18] found lower fractional anisotropy in the superior longitudinal fasciculus and higher fractional anisotropy in the parahippocampal cingulum in individuals with SCD that significantly associated with the functional connectivity of cortical midline structures. Aberrant diffusion characteristics involving physical connections between memory and self-referential regions may distinguish this sample from the others and may reflect a mechanism for lower functional connectivity unrelated to mechanisms underlying increased connectivity [30, 31, 35]. Group differences in diffusion metrics do not explain the group differences in [47] though as there were no differences between the groups in neurite orientation dispersion and density [90] throughout the cingulum fiber tract. However, lower working memory performance for the SCD participants in that sample may have related to decreased connectivity.

Inconsistent results of increased and decreased functional connectivity may also reflect unmeasured confounds. The confounds are unlikely to relate to age as the average sample ages do not appear to drive the differences; they averaged between the mid-sixties to early seventies without a clear pattern of younger or older samples corresponding to particular group differences. Thus, the underlying reason for differences in cross-sectional results could reflect an important unmeasured variable. Though aforementioned sample differences in structural and cognitive measures may have accounted for some of the connectivity variability across studies, there is one commonly unreported variable which may capture a substantial portion of connectivity variance in SCD: time since the onset of perceived cognitive worsening.
Time since onset of SCD may be an important measure for both researchers and clinicians if brain change trajectories over the progression of SCD to objective decline are nonlinear. This suggests a possible reconciliation to the discrepancies of previous connectivity research in SCD. The results may not disprove each other, but rather, they may capture different stages of SCD; high or low network connectivity could reflect different phases of the underlying mechanisms responsible for the experience. Though speculative, and requiring longitudinal evaluation for verification, we propose that elevated network functional connectivity characterizes early SCD, which transitions to lower functional connectivity with time (see Fig. 1). Though, the time course of this nonlinear trajectory may emerge in some regions earlier than other, e.g., in regions most susceptible to early neurodegenerative change like the transentorhinal areas in $\mathrm{AD}$ [91]. This concept of elevated connectivity transitioning to lower connectivity is not novel, and reviews of dementia research have proposed this concept before [92]. However, here, we suggest that this trajectory reflects the underlying neurodegenerative process of the transition between cognitively unimpaired aging and dementia, and that SCD is the experience of this process.

Due to potential nonlinearities, the existence of inflection points in the trajectory of brain changes related to pathology could reduce differences between healthy older adults with and without SCD in cross-sectional analyses. This may not be the case for protein aggregates as they may not spontaneously clear. But for brain measures, such as functional integration, where either a positive or negative association with SCD could be meaningful, and where a change in the direction of association may be equally or more meaningful, the omission of variables related to length of time that participants have experienced SCD may pose a fundamental problem for data analyses. Because of possible nonlinearities in brain changes or other changes related to SCD, longitudinal analysis must drive the discussion regarding potential neurodegenerative-related brain changes in this population. Furthermore, ongoing longitudinal data collection efforts should retroactively gather years-sinceonset information when possible, and new projects should collect this information from the start.

Although a nonlinear trajectory for functional connectivity change makes cross-sectional analysis difficult to interpret, there may be an unseen benefit to this potential change trajectory. Evaluation of an individual over multiple timepoints could help determine their progression though SCD, regardless of initial measurement values. For example, an individual at an early phase of SCD might exhibit increasing functional connectivity over time while an individual on the cusp of converting 


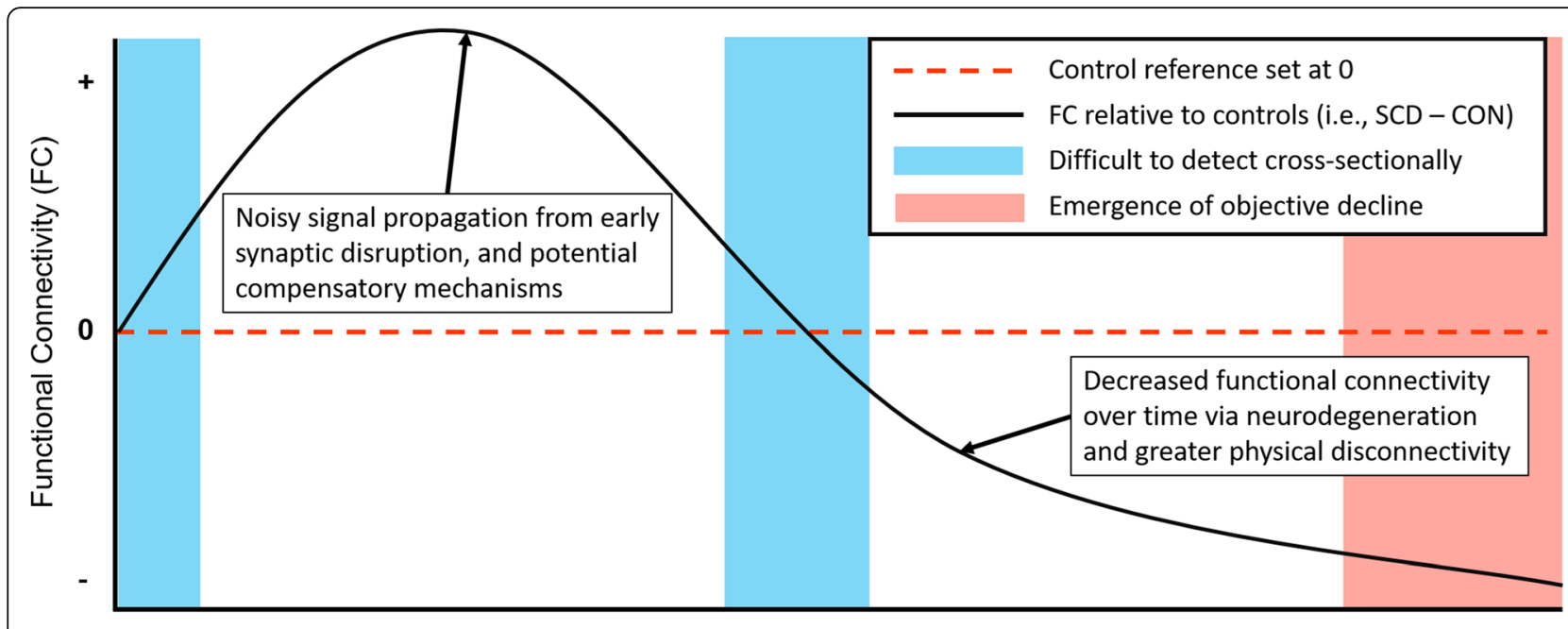

Time since onset of subjective cognitive decline

Fig. 1 Possible trajectory of resting-state functional connectivity changes in subjective cognitive decline for default mode network and medial temporal regions based on cross-sectional analyses. Observations of both greater and lower functional connectivity in SCD could suggest a nonlinear change trajectory over the progression from subjective to objective impairment, highlighting the need for longitudinal analysis. Early synaptic disruption may lead to noisy signal propagation across systems or early compensatory processes that underlie elevated functional connectivity which may transition to lower functional connectivity as neurodegeneration progresses and objective decline become more apparent. Furthermore, this trajectory might shift depending on the brain regions evaluated as some groups report complex patterns of both higher and lower functional connectivity in SCD

to mild cognitive impairment or dementia may exhibit decreasing connectivity over the time. This hypothetical trajectory requires confirmation, but nevertheless suggests that functional connectivity may be a valid method to track the progression of incipient neurodegenerative disease and may be useful regardless of the magnitude of the baseline measurement.

Finally, recruitment methods may also influence functional connectivity results. Perhaps individuals who are concerned about their cognitive ability and seek medical advice represent older adults with SCD more likely to harbor incipient neurodegeneration while individuals recruited from the general public may include SCD cases in an early disease phase, or cases due to neuroticism, poor sleep, or transient stressful life events. The extant literature suggests that recruitment methods may indeed contribute to SCD sample characteristics, with cohorts recruited from memory clinics exhibiting poorer neuropsychological performance, greater hippocampal atrophy, greater cortical atrophy over time, and greater depression than cohorts recruited from the general population that have not sought medical help [93-95]. However, as samples that have sought medical help have exhibited both increased and decreased functional connectivity cross-sectionally $[18,30,47]$, the impact of the severity of cognitive complaint on functional metrics is not as clear. Future research should aim to further clarify if recruitment methodology is an important predictor of the type of functional connectivity pattern exhibited in SCD.

\section{Hypothetical functional model of SCD}

As individuals with SCD may revert to a subjectively normal state, maintain cognitive performance over time, or develop dementia, models of SCD that reflect functional connectivity change related to progressive neurodegeneration implicitly refer to the latter group. While SCD outcomes are heterogenous, we argue that functional neuroimaging has promise as a marker for incipient neurodegeneration and for progression of cognitive decline, distinguishing between those that develop dementia and those that do not. Therefore, we propose the following model to account for common features of SCD based on the aforementioned functional neuroimaging results (Fig. 2).

The first node of the model represents age-related neurodegeneration; at the cellular level, synapse loss could occur before appreciable cell death. Thus, in early SCD, this node may not represent cell loss, but rather physical disconnectivity. This disconnectivity could impact short-range connection within a region or could impact long-range connections that mediate the signaling between different whole-brain networks. For SCD, we speculate that disruptions occur in the hippocampus and surrounding medial temporal lobe through observations of altered task-related hippocampal activity and resting connectivity $[38,43,50]$ and, although not the 


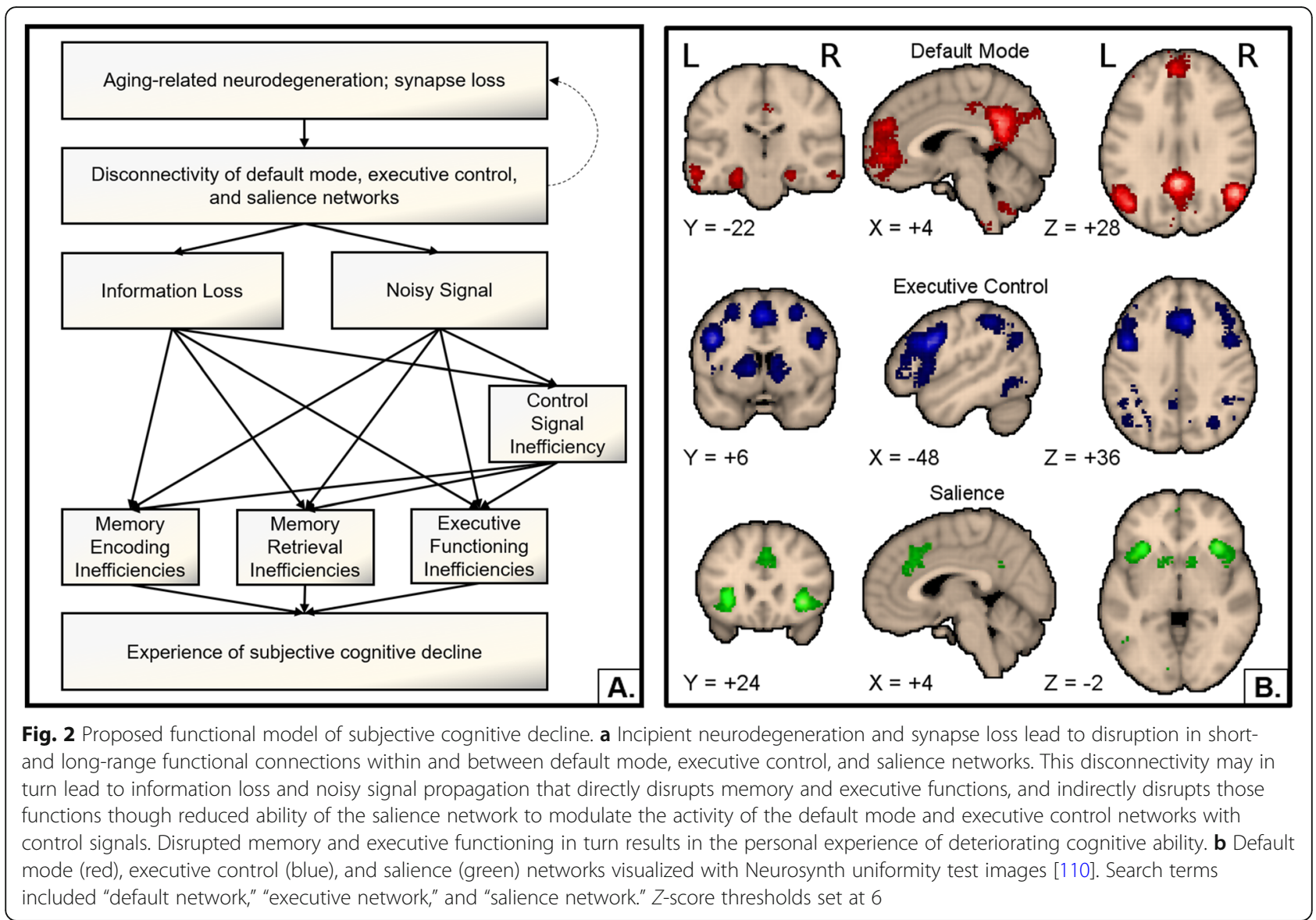

focus of this review, observations of structural atrophy [79-86]. As hippocampal atrophy is a common feature of $\mathrm{AD}$, this may help identify adults with $\mathrm{SCD}$ that could develop $\mathrm{AD}$ specifically while other neurodegenerative patterns could indicate dementia development due to a different cause. We also speculate that long-range connectivity alterations occur between regions of the default mode, executive control, and salience networks based on diffuse task-related and resting functional connectivity alterations across studies $[18,26,30,31,35,38,41-43,47,50]$.

Altered connectivity, both at the local and global level, presumably carries two major consequences: (1) information loss from signal not reaching downstream targets in a global circuit, and (2) noisy signals that reach downstream targets, but arise due to inadequate processing and refinement from local circuits and whole-brain networks. Information loss and noisy signal could lead to processing inefficiencies in control signals from the salience network that mediate memory and executive processes, as well as direct processing inefficiencies in memory encoding or retrieval, and executive functioning. Specific processing inefficiencies may be highly individualized, and the ordering of component failure or regional decline may differ between individuals, but all processing inefficiencies may be sufficient for the experience of SCD, and subtle objective decline that neuropsychological tests may not be sensitive enough to detect. Functional neuroimaging allows for the visualization of these inefficiencies through observation of network functional connectivity and task-related neural activity aberrations, and continued cross-sectional and longitudinal evaluation of network connectivity and activity can help refine this model.

While we place loss of synaptic connections as the first node in the model and speculate that they could have a causal role in disrupting network signaling, it is reasonable to order the events in reverse. Disrupted signaling between brain regions may affect trophic signaling between pre- and post-synaptic neurons and downstream processes related to synaptic maintenance [96, 97], thus having a causal role in neuropil loss. Hebb's postulate stated colloquially: "Cells that fire together, wire together" [98]. Therefore, temporal ordering of events requires exploration, as does the possibility that a positive feedback loop exists where synaptic disconnectivity and gray matter atrophy through neuropil and cell loss amplify functional 
signaling disruption between regions, which in turn exacerbates cell and neuropil loss by disrupting synaptic maintenance processes.

While we emphasize that the proposed and alternative functional models of SCD are non-specific to neurodegenerative etiology and outcome, $\mathrm{AD}$ may be the dominant dementia etiology [19]; thus, it will be important to extend this model, or create a parallel model, to account for individuals with SCD that specifically exhibit AD pathology. As network failure may occur before amyloid- $\beta$ accumulation [20], SCD has variable association with amyloidopathy and tauopathy [11-18], and detection of aberrant amyloid- $\beta$ and tau may precede observable structural decline [99], we speculate that our model could fit in-between the Cascading Network Failure [20] and Pathological Cascade [99] models of AD for older adults with SCD that develop AD specifically. Here, network failure and complex reorganization of processing loads across whole-brain networks could influence metabolic and molecular processes related to $\mathrm{AD}$ pathology and neurodegeneration while simultaneously accounting for the experience of decline. Analyses reporting functional connectivity differences between older adults with and without SCD without finding group differences in amyloid or tau deposition [18, 54] suggest that functional brain alterations and perception of cognitive decline could co-occur before AD pathology detection. Finally, this model could be extended to account for disease-specific outcomes in general; however, future research must determine the patterns of functional connectivity change that are sensitive enough to predict specific outcomes before this model is extended.

\section{Future directions}

While cross-sectional analyses have evaluated default mode and medial temporal functional connectivity, evaluation of other brain networks that SCD may impact remains sparse. Though the medial temporal lobes and default mode network may be sensitive to distinguish SCD from unimpaired, healthy aging, some individuals may display connectivity aberrations in other networks, such as the salience or executive control networks, before default mode network disruption occurs. For example, it is possible that the location of the earliest synaptic disruptions is determined by the specific cognitive domain of the initial complaints. Subjective decline in executive and attentional processing may relate to executive and salience network synaptic disruption prior to memory system disruption. The extant functional neuroimaging literature on SCD largely focuses on memory complaints and does not generally address other cognitive domains perceived as affected. Nevertheless, previous research suggests that connectivity disruption within the salience network, and between the salience network and other networks, may occur in older adults with SCD [43]. Salience network gray matter atrophy occurs at a greater rate in cognitively unimpaired aging than the rest of the brain, and atrophy is even greater in mild cognitive impairment and dementia, and this atrophy may occur alongside lower functional connectivity between salience and executive control network regions and greater connectivity between salience and default mode network regions [100]. If SCD indicates incipient dementia, greater salience network atrophy and connectivity alterations could occur compared to healthy aging and serve as a prognostic marker for further dementia development. In addition, subtle differences in cognitive performance between adults with and without perceived cognitive decline exist [101], and salience network disruption may help explain these group differences, possibly through failure to effectively engage the executive control networks for cognitive tasks while disengaging the default mode network. As mentioned previously, [43] found lower insula activation in individuals with SCD during a task that involved switching between task demands handled by default mode regions and task demands handled by executive control regions. Thus, there is evidence to support exploring connectivity between the default mode, salience, and executive control networks in SCD.

Furthermore, as mentioned earlier, longitudinal analyses must drive the discussion of functional connectivity change trajectories as some groups have observed greater functional connectivity in older adults with $\mathrm{SCD}$ compared to controls while other groups have observed lower functional connectivity. These results suggest a possible nonlinear change in brain network functional integration across the course of SCD. However, the only way to verify that speculation would be extensive longitudinal analysis.

While we emphasize the importance of longitudinal analyses to understand trajectories of functional connectivity change, there are important cross-sectional analyses that could use existing data to help clarify patterns of functional connectivity in SCD. However, they would require coordination across research groups. Currently, a meta-analysis of the functional neuroimaging literature in SCD is infeasible due to too many methodological inconsistencies (e.g., different network definitions, preprocessing pipelines, type of functional connectivity metric utilized, specific regions reported or evaluated). However, if research groups interested in SCD coordinated a common preprocessing and analysis pipeline to evaluate connectivity within and between whole-brain networks, the results could be combined in a pseudometa-analytic framework.

In addition to underexplored network connectivity in $\mathrm{SCD}$, the full extent of functional differences during 
cognitive tasks between older adults with and without perceived decline remains unknown, and the field has yet to report on many detailed components of memory and other cognitive domains through functional neuroimaging. For example, although the extant literature to date has explored memory encoding and retrieval through visual and verbal means, these tasks have lacked explicit spatial components, and some spatial memory processing occurs in the medial temporal lobes [102]. Evaluation of spatial memory encoding and retrieval may be sensitive to detect hippocampal and medial temporal alterations in SCD that may serve as markers of further decline. Furthermore, continued evaluation of memory encoding and retrieval, working memory, and attention is necessary to understand the neural underpinnings of SCD.

Finally, although current neuroimaging resolutions only allow for macro-level evaluation of whole-brain network integration, it is still important to speculate on circuit-level changes that may occur in SCD as information processing ultimately occurs at the synaptic level. The circuitry of the medial temporal lobes may be important for understanding SCD for a multitude of reasons, including previously mentioned connectivity aberrations [30, 35, 38, 47], but also because T1weighted and diffusion-weighted imaging analyses indicate that neuropil density and gray matter volume may be lower in entorhinal, perirhinal, parahippocampal, and hippocampal regions compared to controls [79-86, 103, 104]. Aberrant functional connectivity and significant atrophy within hippocampal circuitry may emerge as appropriate prognostic signals for disease, and the first subfield to critically evaluate may be cornu ammonis 1 as many reports suggest inward deformation of the subfield in older adults with SCD [105-107]. Unfortunately, typical functional connectivity analyses do not have sufficient spatial resolution to determine which hippocampal subfields or which hippocampal and entorhinal cell layers exhibit the greatest differences in connectivity between adults with and without SCD. Even with 2-mm isotropic voxels, subject movement, partial voluming, and spatial smoothing during image processing could smear signal from different hippocampal subregions together, in addition to smearing entorhinal and hippocampal signal. Thus, it is difficult to resolve whether disrupted communication between hippocampus and distant cortex is a downstream result of computation disruption within the hippocampus proper, disruption between direct hippocampus to cortex connections, or disrupted connectivity between entorhinal and parahippocampal cortex and other regions. Nevertheless, highresolution, submillimeter fMRI is undergoing active development and analyses are uncovering functional organization across columnar and laminar cortical structures [108, 109]; this method could resolve aforementioned issues and evaluate hippocampal subfield connectivity. Combination of structural and functional methods could uncover mechanisms underlying SCD. For example, if diffeomorphic mapping finds inward deformation of cornu ammonis 1 in person with SCD that corresponds to decreased connectivity between cornu ammonis 1 and entorhinal cortex through submillimeter imaging, but intact communication between hippocampal subfields, then the results would indicate that hippocampal processing is largely intact but input to-and output from the hippocampus-may be aberrant in incipient neurodegenerative disease. Conversely, intact communication between entorhinal cortex and hippocampal subfields, but aberrant communication between subfields, would indicate that hippocampal processing disruption, rather than input or output disruption, initially occurs. Though speculative, functional neuroimaging in the future may allow for understanding connectivity disruption at the circuit level.

\section{Conclusion}

$\mathrm{SCD}$ is a risk factor for dementia marked by perceived worsening of cognitive ability without observable deficit. The extant task-related activation and resting-state functional connectivity literature suggest that SCD relates to functional alterations in brain regions important for memory, such as retrosplenial cortex and hippocampus. Though, subtle disruption in memory encoding, retrieval, or other cognitive processes may all be sufficient for the experience of cognitive decline. Furthermore, aberrant task-related activation during memory and executive functioning tasks and aberrant functional connectivity in older adults with SCD suggest that information processing alterations may occur in the salience, executive control, and default mode networks. Finally, more research must occur on the functional characteristics of the brain in SCD to understand prognostic markers for dementia, and longitudinal evaluation of functional brain characteristics in SCD must occur specifically to determine trajectories of change that could serve as prognostic and diagnostic markers of objective decline.

\section{Abbreviations \\ AD: Alzheimer's disease; ADNI: Alzheimer's Disease Neuroimaging Initiative; BOLD: Blood-oxygen-level dependent; CON: Control; \\ EEG: Electroencephalography; fMRI: Functional magnetic resonance imaging; MEG: Magnetoencephalography; SCD: Subjective cognitive decline}

\section{Acknowledgements}

Not applicable.

Authors' contributions

$\mathrm{RV}$ and JS contributed to the conceptualization and writing of the manuscript. The authors read and approved the final manuscript 


\section{Availability of data and materials}

Not applicable.

\section{Ethics approval and consent to participate}

Not applicable.

\section{Consent for publication}

Not applicable.

\section{Competing interests}

The authors declare that they have no competing interests.

Received: 1 November 2019 Accepted: 26 February 2020 Published online: 09 March 2020

\section{References}

1. Jessen F, Amariglio RE, van Boxtel M, Breteler M, Ceccaldi M, Chetelat G, et al. A conceptual framework for research on subjective cognitive decline in preclinical Alzheimer's disease. Alzheimers Dement. 2014;10(6):844-52.

2. Mitchell AJ. Is it time to separate subjective cognitive complaints from the diagnosis of mild cognitive impairment? Age Ageing. 2008;37(5):497-9.

3. Gifford KA, Liu DD, Lu ZQ, Tripodis Y, Cantwell NG, Palmisano J, et al. The source of cognitive complaints predicts diagnostic conversion differentially among nondemented older adults. Alzheimers Dement. 2014;10(3):319-27.

4. Jessen F, Wiese B, Bachmann C, Eifflaender-Gorfer S, Haller F, Kolsch H, et al. Prediction of dementia by subjective memory impairment effects of severity and temporal association with cognitive impairment. Arch Gen Psychiat. 2010;67(4):414-22.

5. Jessen F, Wolfsgruber $S$, Wiese B, Bickel H, Mosch E, Kaduszkiewicz H, et al. $\mathrm{AD}$ dementia risk in late $\mathrm{MCl}$, in early $\mathrm{MCl}$, and in subjective memory impairment. Alzheimers Dement. 2014;10(1):76-83.

6. Reisberg B, Shulman MB, Torossian C, Leng L, Zhu W. Outcome over seven years of healthy adults with and without subjective cognitive impairment. Alzheimers Dement. 2010;6(1):11-24.

7. Reisberg B, Prichep L, Mosconi L, John ER, Glodzik-Sobanska L, Boksay I, et al. The pre-mild cognitive impairment, subjective cognitive impairment stage of Alzheimer's disease. Alzheimers Dement. 2008;4(1):S98-S108.

8. Abdulrab K, Heun R. Subjective memory impairment. A review of its definitions indicates the need for a comprehensive set of standardised and validated criteria. Eur Psychiat. 2008;23(5):321-30.

9. Reisberg B, Gauthier S. Current evidence for subjective cognitive impairment (SCI) as the pre-mild cognitive impairment (MCI) stage of subsequently manifest Alzheimer's disease. Int Psychogeriatr. 2008;20(1):1-16.

10. Jack CR, Bennett DA, Blennow K, Carrillo MC, Dunn B, Haeberlein SB, et al. NIA-AA research framework: toward a biological definition of Alzheimer's disease. Alzheimers Dement. 2018;14(4):535-62.

11. Amariglio RE, Becker JA, Carmasin J, Wadsworth LP, Lorius N, Sullivan C, et al. Subjective cognitive complaints and amyloid burden in cognitively normal older individuals. Neuropsychologia. 2012;50(12):2880-6.

12. Amariglio RE, Mormino EC, Pietras AC, Marshall GA, Vannini P, Johnson KA et al. Subjective cognitive concerns, amyloid-beta, and neurodegeneration in clinically normal elderly. Neurology. 2015;85(1):56-62.

13. Buckley RF, Hanseeuw B, Schultz AP, Vannini P, Aghjayan SL, Properzi MJ, et al. Region-specific association of subjective cognitive decline with tauopathy independent of global beta-amyloid burden. JAMA Neurol. 2017; 74(12):1455-63.

14. Kim JW, Byun MS, Yi D, Lee JH, Ko K, Jung G, et al. Vascular risk modulates the relationship between cerebral amyloid deposition and subjective memory complaints. Neuropsych Dis Treat. 2019;15:637-45.

15. Eckerstrom M, Berg Al, Nordlund A, Rolstad S, Sacuiu S, Wallin A. High prevalence of stress and low prevalence of Alzheimer disease CSF biomarkers in a clinical sample with subjective cognitive impairment. Dement Geriatr Cogn Disord. 2016:42(1-2):93-105.

16. Pike KE, Ellis KA, Villemagne VL, Good N, Chetelat G, Ames D, et al. Cognition and beta-amyloid in preclinical Alzheimer's disease: data from the AIBL study. Neuropsychologia. 2011;49(9):2384-90.

17. Hollands S, Lim YY, Buckley R, Pietrzak RH, Snyder PJ, Ames D, et al. Amyloid-beta related memory decline is not associated with subjective or informant rated cognitive impairment in healthy adults. J Alzheimers Dis. 2015;43(2):677-86.
18. Yasuno F, Kazui H, Yamamoto A, Morita N, Kajimoto K, Ihara M, et al. Resting-state synchrony between the retrosplenial cortex and anterior medial cortical structures relates to memory complaints in subjective cognitive impairment. Neurobiol Aging. 2015;36(6):2145-52.

19. van der Flier WM, Scheltens P. Epidemiology and risk factors of dementia. J Neurol Neurosurg Psychiatry. 2005;76(Suppl 5):v2-7.

20. Jones DT, Knopman DS, Gunter JL, Graff-Radford J, Vemuri P, Boeve BF, et al. Cascading network failure across the Alzheimer's disease spectrum. Brain. 2016;139:547-62.

21. Sheline YI, Morris JC, Snyder AZ, Price JL, Yan Z, D'Angelo G, et al. APOE4 allele disrupts resting state $\mathrm{fMRI}$ connectivity in the absence of amyloid plaques or decreased CSF Abeta42. J Neurosci. 2010;30(50):17035-40.

22. Salami A, Wahlin A, Kaboodvand N, Lundquist A, Nyberg L. Longitudinal evidence for dissociation of anterior and posterior MTL resting-state connectivity in aging: links to perfusion and memory. Cereb Cortex. 2016; 26(10):3953-63.

23. Fjell $A M$, Sneve $M H$, Storsve AB, Grydeland $H$, Yendiki A, Walhovd KB. Brain events underlying episodic memory changes in aging: a longitudinal investigation of structural and functional connectivity. Cereb Cortex. 2016; 26(3):1272-86

24. Ye Q, Chen HF, Su F, Shu H, Gong L, Xie CM, et al. An inverse U-shaped curve of resting-state networks in individuals at high risk of Alzheimer's disease. J Clin Psychiat. 2018;79(2):17m11583.

25. Alexander DM, Arns MW, Paul RH, Rowe DL, Cooper N, Esser AH, et al. EEG markers for cognitive decline in elderly subjects with subjective memory complaints. J Integr Neurosci. 2006;5(1):49-74.

26. Rodda JE, Dannhauser TM, Cutinha DJ, Shergill SS, Walker Z. Subjective cognitive impairment: increased prefrontal cortex activation compared to controls during an encoding task. Int J Geriatr Psychiatry. 2009;24(8):865-74.

27. Maestu F, Baykova E, Ruiz JM, Montejo P, Montenegro M, Llanero M, et al. Increased biomagnetic activity in healthy elderly with subjective memory complaints. Clin Neurophysiol. 2011;122(3):499-505.

28. Rodda J, Dannhauser T, Cutinha DJ, Shergill SS, Walker Z. Subjective cognitive impairment: functional MRI during a divided attention task. Eur Psychiatry. 2011;26(7):457-62.

29. Dumas JA, Kutz AM, McDonald BC, Naylor MR, Pfaff AC, Saykin AJ, et al. Increased working memory-related brain activity in middle-aged women with cognitive complaints. Neurobiol Aging. 2013:34(4):1145-7.

30. Hafkemeijer A, Altmann-Schneider I, Oleksik AM, van de Wiel L, Middelkoop HA, van Buchem MA, et al. Increased functional connectivity and brain atrophy in elderly with subjective memory complaints. Brain Connect. 2013; 3(4):353-62.

31. Dillen KNH, Jacobs HIL, Kukolja J, von Reutern B, Richter N, Onur OA, et al. Aberrant functional connectivity differentiates retrosplenial cortex from posterior cingulate cortex in prodromal Alzheimer's disease. Neurobiol Aging. 2016:44:114-26

32. Sun Y, Dai ZJ, Li YX, Sheng C, Li HY, Wang XN, et al. Subjective cognitive decline: mapping functional and structural brain changes-a combined resting-state functional and structural MR imaging study. Radiology. 2016; 281(1):185-92

33. Cespon J, Galdo-Alvarez S, Diaz F. Event-related potentials reveal altered executive control activity in healthy elderly with subjective memory complaints. Front Hum Neurosci. 2018;12:445.

34. Lazarou I, Adam K, Georgiadis K, Tsolaki A, Nikolopoulos S, Kompatsiaris I, et al. Can a novel high-density EEG approach disentangle the differences of visual event related potential (N170), elicited by negative facial stimuli, in people with subjective cognitive impairment? J Alzheimers Dis. 2018;65(2):543-75.

35. Verfaillie SCI, Pichet Binette A, Vachon-Presseau E, Tabrizi S, Savard M, Bellec P, et al. Subjective cognitive decline is associated with altered default mode network connectivity in individuals with a family history of Alzheimer's disease. Biol Psychiatry Cogn Neurosci Neuroimaging. 2018;3(5):463-72.

36. Kawagoe T, Onoda K, Yamaguchi S. Subjective memory complaints are associated with altered resting-state functional connectivity but not structural atrophy. Neuroimage-Clin. 2019;21:101675.

37. Bajo R, Castellanos NP, Lopez ME, Ruiz JM, Montejo P, Montenegro M, et al. Early dysfunction of functional connectivity in healthy elderly with subjective memory complaints. Age. 2012;34(2):497-506.

38. Wang Y, Risacher SL, West JD, McDonald BC, Magee TR, Farlow MR, et al. Altered default mode network connectivity in older adults with cognitive complaints and amnestic mild cognitive impairment. J Alzheimers Dis. 2013 35(4):751-60. 
39. Lopez-Sanz D, Bruna R, Garces P, Camara C, Serrano N, Rodriguez-Rojo IC, et al. Alpha band disruption in the AD-continuum starts in the subjective cognitive decline stage: a MEG study. Sci Rep-Uk. 2016;6:37685.

40. Contreras JA, Goni J, Risacher SL, Amico E, Yoder K, Dzemidzic M, et al. Cognitive complaints in older adults at risk for Alzheimer's disease are associated with altered resting-state networks. Alzheimers Dement (Amst). 2017;6:40-9.

41. Dillen KNH, Jacobs HIL, Kukolja J, Richter N, von Reutern B, Onur OA, et al. Functional disintegration of the default mode network in prodromal Alzheimer's disease. J Alzheimers Dis. 2017;59(1):169-87.

42. Hayes JM, Tang LF, Viviano RP, van Rooden S, Ofen N, Damoiseaux JS. Subjective memory complaints are associated with brain activation supporting successful memory encoding. Neurobiol Aging. 2017;60:71-80.

43. Hu X, Uhle F, Fliessbach K, Wagner M, Han Y, Weber B, et al. Reduced future-oriented decision making in individuals with subjective cognitive decline: a functional MRI study. Alzheimers Dement (Amst). 2017;6:222-31.

44. Mazzon G, De Dea F, Cattaruzza T, Manganotti P, Monti F, Accardo A. Memorization test and resting state EEG components in mild and subjective cognitive impairment. Curr Alzheimer Res. 2018; 15(9):809-19.

45. Yang L, Yan Y, Wang YH, Hu XC, Lu J, Chan P, et al. Gradual disturbances of the amplitude of low-frequency fluctuations (ALFF) and fractional ALFF in Alzheimer spectrum. Front Neurosci-Switz. 2018;12:975.

46. Lopez-Sanz D, Bruna R, Delgado-Losada ML, Lopez-Higes R, Marcos-Dolado A, Maestu F, et al. Electrophysiological brain signatures for the classification of subjective cognitive decline: towards an individual detection in the preclinical stages of dementia. Alzheimers Res Ther. 2019;11:49.

47. Viviano RP, Hayes JM, Pruitt PJ, Fernandez ZJ, van Rooden S, van der Grond J, et al. Aberrant memory system connectivity and working memory performance in subjective cognitive decline. Neuroimage. 2019;185:556-64.

48. Wang ZQ, Qiao KN, Chen GQ, Sui DY, Dong HM, Wang YS, et al. Functional connectivity changes across the spectrum of subjective cognitive decline, amnestic mild cognitive impairment and Alzheimer's disease. Front Neuroinformatics. 2019:13:26.

49. Babiloni C, Visser PJ, Frisoni G, De Deyn PP, Bresciani L, Jelic V, et al. Cortical sources of resting EEG rhythms in mild cognitive impairment and subjective memory complaint. Neurobiol Aging. 2010;31(10):1787-98.

50. Erk S, Spottke A, Meisen A, Wagner M, Walter H, Jessen F. Evidence of neuronal compensation during episodic memory in subjective memory impairment. Arch Gen Psychiat. 2011;68(8):845-52.

51. Vega JN, Zurkovsky L, Albert K, Melo A, Boyd B, Dumas J, et al. Altered brain connectivity in early postmenopausal women with subjective cognitive impairment. Front Neurosci-Switz. 2016;10:433.

52. Lopez-Sanz D, Bruna R, Garces P, Martin-Buro MC, Walter S, Delgado ML, et al. Functional connectivity disruption in subjective cognitive decline and mild cognitive impairment: a common pattern of alterations. Front Aging Neurosci. 2017;9:109.

53. Jiang LL, Sui DY, Qiao KN, Dong HM, Chen LN, Han Y. Impaired functional criticality of human brain during Alzheimer's disease progression. Sci RepUk. 2018;8:1-11.

54. Li KC, Luo X, Zeng QZ, Jiaerken Y, Xu XJ, Huang PY, et al. Aberrant functional connectivity network in subjective memory complaint individuals relates to pathological biomarkers. Translational Neurodegeneration. 2018;7:1-10.

55. Xie $Y Y$, Liu TT, Ai J, Chen DD, Zhuo YR, Zhao GL, et al. Changes in centrality frequency of the default mode network in individuals with subjective cognitive decline. Front Aging Neurosci. 2019;11:118.

56. Yan TY, Wang YH, Weng ZZ, Du WY, Liu TT, Chen DD, et al. Early-stage identification and pathological development of Alzheimer's disease using multimodal MRI. J Alzheimers Dis. 2019;68(3):1013-27.

57. Dierks T, Jelic V, Julin P, Maurer K, Wahlund LO, Almkvist O, et al. EEGmicrostates in mild memory impairment and Alzheimer's disease: possible association with disturbed information processing. J Neural Transm. 1997; 104(4-5):483-95.

58. Lopez-Sanz D, Garces P, Alvarez B, Delgado-Losada ML, Lopez-Higes R Maestu F. Network disruption in the preclinical stages of Alzheimer's disease: from subjective cognitive decline to mild cognitive impairment. Int J Neural Syst. 2017;27(8):1750041

59. Teipel SJ, Metzger CD, Brosseron F, Buerger K, Brueggen K, Catak C, et al Multicenter resting state functional connectivity in prodromal and dementia stages of Alzheimer's disease. J Alzheimers Dis. 2018;64(3):801-13.

60. Contreras JA, Avena-Koenigsberger A, Risacher SL, West JD, Tallman E, $\mathrm{McD}$ onald $\mathrm{BC}$, et al. Resting state network modularity along the prodromal late onset Alzheimer's disease continuum. Neuroimage-Clin. 2019;22:101687.
61. Scarapicchia V, Garcia-Barrera M, MacDonald S, Gawryluk JR. Resting state BOLD variability is linked to white matter vascular burden in healthy aging but not in older adults with subjective cognitive decline. Front Hum Neurosci. 2019:13:429.

62. Balash Y, Mordechovich M, Shabtai H, Giladi N, Gurevich T, Korczyn AD. Subjective memory complaints in elders: depression, anxiety, or cognitive decline? Acta Neurol Scand. 2013;127(5):344-50.

63. de Guzman AB, Lagdaan LFM, Lagoy MLV. The role of life-space, social activity, and depression on the subjective memory complaints of community-dwelling Filipino elderly: a structural equation model. Educ Gerontol. 2015;41(5):348-60.

64. Montejo P, Montenegro M, Fernandez MA, Maestu F. Subjective memory complaints in the elderly: prevalence and influence of temporal orientation, depression and quality of life in a population-based study in the city of Madrid. Aging Ment Health. 2011;15(1):85-96.

65. Plotkin DA, Mintz J, Jarvik LF. Subjective memory complaints in geriatric depression. Am J Psychiat. 1985;142(9):1103-5.

66. Reid LM, MacLullich AMJ. Subjective memory complaints and cognitive impairment in older people. Dement Geriatr Cogn. 2006;22(5-6):471-85.

67. Ponds RWHM, Jolles J. Memory complaints in elderly people: the role of memory abilities, metamemory, depression, and personality. Educ Gerontol. 1996;22(4):341-57.

68. Slavin MJ, Brodaty H, Kochan NA, Crawford JD, Trollor JN, Draper B, et al. Prevalence and predictors of "subjective cognitive complaints" in the Sydney memory and ageing study. Am J Geriat Psychiat. 2010;18(8): 701-10.

69. Buckner RL, Andrews-Hanna JR, Schacter DL. The brain's default network anatomy, function, and relevance to disease. Year in Cognitive Neuroscience 2008. 2008;1124:1-38.

70. Seeley WW, Menon V, Schatzberg AF, Keller J, Glover GH, Kenna H, et al. Dissociable intrinsic connectivity networks for salience processing and executive control. J Neurosci. 2007;27(9):2349-56.

71. Dosenbach NUF, Fair DA, Miezin FM, Cohen AL, Wenger KK, Dosenbach RAT, et al. Distinct brain networks for adaptive and stable task control in humans. P Natl Acad Sci USA. 2007;104(26):11073-8.

72. Menon V, Uddin LQ. Saliency, switching, attention and control: a network model of insula function. Brain Struct Funct. 2010;214(5-6):655-67.

73. Logothetis NK, Pauls J, Augath M, Trinath T, Oeltermann A. Neurophysiological investigation of the basis of the fMRI signal. Nature. 2001;412(6843):150-7.

74. Park DC, Reuter-Lorenz P. The adaptive brain: aging and neurocognitive scaffolding. Annu Rev Psychol. 2009;60:173-96.

75. Chan MY, Park DC, Savalia NK, Petersen SE, Wig GS. Decreased segregation of brain systems across the healthy adult lifespan. Proc Natl Acad Sci U S A. 2014;111(46):E4997-5006.

76. Goh JOS. Functional dedifferentiation and altered connectivity in older adults: neural accounts of cognitive aging. Aging Dis. 2011;2(1):30-48.

77. Morcom AM, Henson RNA. Increased prefrontal activity with aging reflects nonspecific neural responses rather than compensation. J Neurosci. 2018; 38(33):7303-13.

78. Morcom AM, Li J, Rugg MD. Age effects on the neural correlates of episodic retrieval: increased cortical recruitment with matched performance. Cereb Cortex. 2007:17(11):2491-506.

79. Kim MJ, Seo SW, Kim GH, Kim ST, Lee JM, Qiu AQ, et al. Less depressive symptoms are associated with smaller hippocampus in subjective memory impairment. Arch Gerontol Geriat. 2013;57(1):110-5.

80. Striepens N, Scheef L, Wind A, Popp J, Spottke A, Cooper-Mahkorn D, et al. Volume loss of the medial temporal lobe structures in subjective memory impairment. Dement Geriatr Cogn. 2010;29(1):75-81.

81. Scheef L, Spottke A, Daerr M, Joe A, Striepens N, Kolsch H, et al. Glucose metabolism, gray matter structure, and memory decline in subjective memory impairment. Neurology. 2012;79(13):1332-9.

82. Yue L, Wang T, Wang JY, Li GJ, Wang JH, Li X, et al. Asymmetry of hippocampus and amygdala defect in subjective cognitive decline among the community dwelling Chinese. Front Psychiatry. 2018;9:226.

83. Stewart R, Dufouil C, Godin O, Ritchie K, Maillard P, Delcroix N, et al. Neuroimaging correlates of subjective memory deficits in a community population. Neurology. 2008;70(18):1601-7.

84. Jessen F, Feyen L, Freymann K, Tepest R, Maier W, Heun R, et al. Volume reduction of the entorhinal cortex in subjective memory impairment. Neurobiol Aging. 2006;27(12):1751-6. 
85. Fan LY, Lai YM, Chen TF, Hsu YC, Chen PY, Huang KZ, et al. Diminution of context association memory structure in subjects with subjective cognitive decline. Hum Brain Mapp. 2018;39(6):2549-62.

86. van der Flier WM, van Buchem MA, Weverling-Rijnsburger AWE, Mutsaers ER, Bollen ELEM, Admiraal-Behloul F, et al. Memory complaints in patients with normal cognition are associated with smaller hippocampal volumes. Neurol. 2004;251(6):671-5.

87. Gallagher M, Bakker A, Yassa MA, Stark CEL. Bridging neurocognitive aging and disease modification: targeting functional mechanisms of memory impairment. Curr Alzheimer Res. 2010;7(3):197-9.

88. Northoff G, Heinzel A, Greck M, Bennpohl F, Dobrowolny H, Panksepp J. Self-referential processing in our brain - a meta-analysis of imaging studies on the self. Neuroimage. 2006;31(1):440-57.

89. Ranganath C, Ritchey M. Two cortical systems for memory-guided behaviour. Nat Rev Neurosci. 2012;13(10):713-26.

90. Zhang H, Schneider T, Wheeler-Kingshott CA, Alexander DC. NODDI: practical in vivo neurite orientation dispersion and density imaging of the human brain. Neuroimage. 2012;61(4):1000-16.

91. Braak H, Braak E. Neuropathological staging of Alzheimer-related changes. Acta Neuropathol. 1991;82(4):239-59.

92. Sperling RA, Dickerson BC, Pihlajamaki M, Vannini P, LaViolette PS, Vitolo OV, et al. Functional alterations in memory networks in early Alzheimer's disease. NeuroMolecular Med. 2010;12(1):27-43.

93. Abdelnour C, Rodriguez-Gomez O, Alegret M, Valero S, Moreno-Grau S, Sanabria A, et al. Impact of recruitment methods in subjective cognitive decline. J Alzheimers Dis. 2017;57(2):625-32.

94. Perrotin A, La Joie R, de La Sayette V, Barre L, Mezenge F, Mutlu J, et al. Subjective cognitive decline in cognitively normal elders from the community or from a memory clinic: differential affective and imaging correlates. Alzheimers Dement. 2017;13(5):550-60.

95. Kuhn E, Moulinet I, Perrotin A, La Joie R, Landeau B, Tomadesso C, et al. Cross-sectional and longitudinal characterization of SCD patients recruited from the community versus from a memory clinic: subjective cognitive decline, psychoaffective factors, cognitive performances, and atrophy progression over time. Alzheimers Res Ther. 2019;11(1):61.

96. Lin YC, Koleske AJ. Mechanisms of Synapse and Dendrite Maintenance and Their Disruption in Psychiatric and Neurodegenerative Disorders. Ann Rev Neurosci. 2010;33:349-78.

97. Benson TE, Ryugo DK, Hinds JW. Effects of sensory deprivation on the developing mouse olfactory system - a light and electron-microscopic, morphometric analysis. J Neurosci. 1984;4(3):638-53.

98. Hebb DO. Organization of behavior. J Clin Psychol. 1950;6(3):307.

99. Jack CR, Knopman DS, Jagust WJ, Petersen RC, Weiner MW, Aisen PS, et al. Tracking pathophysiological processes in Alzheimer's disease: an updated hypothetical model of dynamic biomarkers. Lancet Neurol. 2013;12(2):207-16.

100. He XX, Qin W, Liu Y, Zhang XQ, Duan YY, Song JY, et al. Abnormal salience network in normal aging and in amnestic mild cognitive impairment and Alzheimer's disease. Hum Brain Mapp. 2014;35(7):3446-64.

101. Burmester B, Leathem J, Merrick P. Subjective cognitive complaints and objective cognitive function in aging: a systematic review and meta-analysis of recent cross-sectional findings. Neuropsychol Rev. 2016;26(4):376-93.

102. Ryan L, Lin CY, Ketcham K, Nadel L. The role of medial temporal lobe in retrieving spatial and nonspatial relations from episodic and semantic memory. Hippocampus. 2010;20(1):11-8.

103. Meiberth D, Scheef L, Wolfsgruber S, Boecker H, Block W, Traber F, et al. Cortical thinning in individuals with subjective memory impairment. J Alzheimers Dis. 2015;45(1):139-46.

104. Cherbuin N, Sargent-Cox K, Easteal S, Sachdev P, Anstey KJ. Hippocampal atrophy is associated with subjective memory decline: the PATH through life study. Am J Geriat Psychiat. 2015;23(5):446-55.

105. Perrotin A, de Flores R, Lamberton F, Poisnel G, La Joie R, de la Sayette V, et al. Hippocampal subfield volumetry and 3D surface mapping in subjective cognitive decline. J Alzheimers Dis. 2015;48:S141-S50.

106. Cantero JL, Iglesias JE, Van Leemput K, Atienza M. Regional hippocampal atrophy and higher levels of plasma amyloid-beta are associated with subjective memory complaints in nondemented elderly subjects. J Gerontol a-Biol. 2016;71(9):1210-5.

107. Tepest R, Wang L, Csernansky JG, Neubert P, Heun R, Scheef L, et al. Hippocampal surface analysis in subjective memory impairment, mild cognitive impairment and Alzheimer's dementia. Dement Geriatr Cogn. 2008;26(4):323-9.
108. Huber L, Handwerker DA, Jangraw DC, Chen G, Hall A, Stuber C, et al. Highresolution CBV-fMRI allows mapping of laminar activity and connectivity of cortical input and output in human M1. Neuron. 2017;96(6):1253-63 e7.

109. Goense J, Merkle H, Logothetis NK. High-resolution fMRI reveals laminar differences in neurovascular coupling between positive and negative BOLD responses. Neuron. 2012;76(3):629-39.

110. Yarkoni T, Poldrack RA, Nichols TE, Van Essen DC, Wager TD. Large-scale automated synthesis of human functional neuroimaging data. Nat Methods. 2011;8(8):665-U95.

\section{Publisher's Note}

Springer Nature remains neutral with regard to jurisdictional claims in published maps and institutional affiliations. 